\title{
OCCUPATIONAL EXPOSURE TO ASBESTOS AND CHOLANGIOCARCINOMA: FINDINGS FROM AN EXPLORATIVE CASE-CONTROL ANALYSIS
}

Giovanni Brandi, Andrea Farioli, Stefania Di Girolamo, Stefania Curti, Francesco De Rosa, Francesco Violante, Guido Biasco, Stefano Mattioli University of Bologna, Bologna, Italy

10.1136/oemed-2011-100382.66

Objectives Alongside to respiratory cancers, exposure to asbestos has been associated with gastrointestinal cancers. An association between exposure to amphibole fibres and their presence in hepatic tissue has been reported. We conducted a case-control analysis to explore the association between occupational exposure to asbestos and cholangiocarcinoma.

Methods We used historical data from 155 consecutive patients referred to our centre for diagnosis and treatment of cholangiocarcinoma in 2007-2011 (69 affected by Intrahepatic Cholangiocarcinoma (ICC) and 86 by Extrahepatic Cholangiocarcinoma (ECC)). When feasible, cases were individually matched (ratio up to 1:4) by calendar period of birth (5-year interval), sex and provenience to historical population controls (originally sampled to study other occupational diseases, not related to asbestos). Occupational exposure to asbestos was assessed by industrial hygienists considering life-prevalent job titles. Separate conditional logistic regression models were conducted for ICC and ECC, adjusting for smoking status and socioeconomic class.

Results We matched 149 controls to 49 cases of ICC and 212 controls to 59 cases of ECC (47 cases were not matched mainly due to provenience). We found an increased risk of ICC in workers exposed to asbestos (adjusted OR 4.73, 95\% CI 1.54 to 14.54); conversely, no clear evidence of increased risk was found for ECC (adjusted OR 1.84, 95\% CI 0.66 to 5.08). Sensitivity analysis conducted using only patients from our city district (conducted to minimise referral bias) produced confirmatory figures.

Conclusions Findings from our exploratory study support the hypothesis that intrahepatic cholangiocarcinoma could arise from chronic irritation and inflammation caused by deposition of asbestos fibres. 